\title{
Structure and conductance histogram of atomic-sized Au contacts
}

\author{
M. Dreher, ${ }^{1}$ F. Pauly, ${ }^{2}$ J. Heurich,${ }^{2}$ J.C. Cuevas, ${ }^{2}$ E. Scheer,${ }^{1}$ and P. Nielaba ${ }^{1}$ \\ ${ }^{1}$ Physics Department, University of Konstanz, 78457 Konstanz, Germany \\ ${ }^{2}$ Institut für Theoretische Festkörperphysik, University of Karlsruhe, 76128 Karlsruhe, Germany
}

(Dated: February 2, 2008)

\begin{abstract}
Many experiments have shown that the conductance histograms of metallic atomic-sized contacts exhibit a peak structure, which is characteristic of the corresponding material. The origin of these peaks still remains as an open problem. In order to shed some light on this issue, we present a theoretical analysis of the conductance histograms of Au atomic contacts. We have combined classical molecular dynamics simulations of the breaking of nanocontacts with conductance calculations based on a tight-binding model. This combination gives us access to crucial information such as contact geometries, forces, minimum cross-section, total conductance and transmission coefficients of the individual conduction channels. The ensemble of our results suggests that the low temperature $\mathrm{Au}$ conductance histograms are a consequence of a subtle interplay between mechanical and electrical properties of these nanocontacts. At variance with other suggestions in the literature, our results indicate that the peaks in the Au conductance histograms are not a simple consequence of conductance quantization or the existence of exceptionally stable radii. We show that the main peak in the histogram close to one quantum of conductance is due to the formation of single-atom contacts and chains of gold atoms. Moreover, we present a detailed comparison with experimental results on $\mathrm{Au}$ atomic contacts where the individual channel transmissions have been determined.
\end{abstract}

PACS numbers: 73.63.Rt, 73.40.Jn, 05.10.-a, 02.70.Ns

\section{INTRODUCTION}

The metallic nanowires fabricated by means of the scanning-tunneling microscope and break-junction techniques have turned out to be an unique playground where to test concepts on electronic transport at the atomic

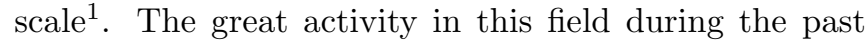
decade was triggered by the observation of a step-like evolution of the conductance during the formation of the nanowires ${ }^{2.3}$. Immediately, the connection with conductance quantization (CQ) observed in two-dimensional electron-gas devices ${ }^{4}$ was discussed, where every conduction channel has a perfect transparency. In order to investigate in detail CQ, different authors introduced conductance histograms, constructed from a large number of individual conductance curves ${ }^{5.6 .7}$, i.e. the evolution of the conductance with the elongation. These demonstrated that for metals like gold and sodium the conductance has a certain preference to adopt multiples of $G_{0}=2 e^{2} / h$, the quantum of conductance. However, for a large variety of metals, the peaks in the histograms do not appear at multiples of $G_{0}$ (for a detailed discussion of the conductance histograms, see section V.D in Ref. [1]). For instance, it has been shown ${ }^{8}$ that an $\mathrm{Al}$ contact sustains up to three conduction channels in the last conductance plateau, although the typical conductance is close to $1 G_{0}$. The $\mathrm{Al}$ histogram exhibits a series of peaks ${ }^{9}$, which in view of the results of Ref. [8] cannot be interpreted as CQ. These results showed that one cannot simply interpret the peaks close to multiples of $G_{0}$ in the histograms as evidence for $\mathrm{CQ}^{9}$. The results of Ref. [8] were explained on the basis of a tight-binding mode10.11. From this work the picture that emerged was that the number of conduction channels is limited by the valence orbitals of the corresponding metal, and the channels are in general not fully transparent. This view was confirmed experimentally by a systematic study for various metals $\frac{12}{2}$. In spite of the success of this atomic picture, the origin of the peaks in the conductance histograms remains unclear, and the main goal of this work is to shed some light on this issue.

It is obvious that the mechanical properties of the nanowires can play an important role in the shape of a conductance histogram. This was pointed out in early molecular-dynamics simulations 13.14 .15 , which suggested that the steps in the conductance during the formation of the nanowires are due to atomic rearrangements. Clear experimental evidence of this fact was reported by Rubio et al.$\frac{16}{}$, who combined conductance and force measurements to show that the jumps in the conductance are associated with distinct jumps in the force. A striking example of the interplay between mechanical and electrical properties can be found in the work of Yanson et $a l \stackrel{17.18}{~}$. These authors observed a series of peaks in the histograms of alkali metals at relative high temperatures, which were convincingly interpreted as the existence of exceptionally stable contact diameters due to electronic and atomic shell effects. This confirmed the idea that the quantum modes in nanowires not only determine the conductance but also play an important role in the cohesive energy 19.20.21. More recently, Hasmy et al $\stackrel{22}{\underline{22}}$ have calculated histograms of the minimum cross-section for $\mathrm{Al}$ contacts using molecular dynamics. At low temperatures they obtained peaks at multiples of the cross-section of a single atom, which led them to a new interpretation of the conductance histogram peaks based on preferential geometrical arrangements of nanocontact necks. It is also important to mention that very recently the room temperature conductance histograms of $\mathrm{Au}$ and $\mathrm{Al}$ have 
been interpreted as an evidence of electronic and atomic shell effect in these metals 23.24 .

So far, several publications have analysed the evolution of metallic nanowires in a stretching event ${ }^{25.26}$. The theoretical analysis of conductance histograms, however, has only been carried out within the framework of freeelectron models by choosing particular nanowire dynam$\operatorname{ics}^{27}$. Some authors have combined classical moleculardynamics simulations with atomistic calculations of the conductance ${ }^{15.28,29.30 .31 .32 .33 .34}$ to analyze single realizations of the contact. The existence of some exceptionally stable structures and the correlation of conductance histograms and atomic structure were previously published for gold using the Wulff-construction method and the Hückel approximation for the conductance 35.36 . However, there are no full atomistic studies of the conductance histograms.

In this work we report for the first time a full atomistic study of the conductance histograms of gold atomic contacts, and we present an extensive comparison with experimental results. For this purpose, we have combined atomistic calculations of the conductance within a tight-binding approximation with realistic molecular dynamics simulations of contact geometries. This combination allows us to obtain detailed information on the mechanical and transport properties such as contact geometries, forces, minimum cross-section, total conductance, the number and the evolution of individual transmission coefficients. We have chosen the element gold for two reasons. First, gold histograms have been widely investigated 5.6 .37 .38 .39 .40 .41 . One typically observes a pronounced peak at $1 G_{0}$, and much smaller peaks close to $2 G_{0}$ and $3 G_{0}$, although the histograms slightly depend on details such as temperature, voltage bias and vacuum conditions. Second, the experimental analysis of the individual conduction channels using the technique introduced in Ref. [8] is available ${ }^{12,42,43,44}$. This analysis shows that conduction channels disappear one by one as the contact is elongated, and in the last plateau, which corresponds to either a single-atom contact or a chain 45.46 , the conductance is dominated by a single channel.

Our results show that the histograms of the minimum cross-section at low temperatures $(4 \mathrm{~K})$ exhibit peaks, suggesting the existence of some exceptionally stable structures. From a semi-classical point of view, one would then expect these peaks to be reflected as peaks in the conductance histograms. However, our results indicate that this is in general not the case. Indeed, only in the last stage of the stretching process we obtain a large peak close to $1 G_{0}$, which is correlated to the formation of a single-atom contact or a chain of atoms. Our channel analysis shows that the conduction modes disappear one by one and the last plateau is dominated by a single channel, in agreement with the experimental results. In short, the ensemble of our results shows that the low-temperature conductance histograms of gold are a consequence of the interplay between the mechanical and the electrical properties of these atomic contacts.

The rest of the paper is organized as follows. In section II we explain the technical details of our molecular dynamics simulations and conductance calculations. Section III is devoted to the analysis of some examples of the breaking of the contacts, where in particular we show the evolution of the single channels and illustrate the typical geometries formed in the last stages: single-atom contacts and chains of atoms. In section IV we present new experimental results of the determination of the channel transmissions in Au contacts and we compare these results with our theoretical simulations. In section $\mathrm{V}$ we discuss our results for the histograms of the minimum cross-section. The corresponding results for the conductance histogram are presented in section VI and their correlation with the minimum cross-section histograms is discussed in detail. Finally, in section VII we present a brief summary of the main results and conclusions.

\section{DESCRIPTION OF THE THEORETICAL MODEL}

As mentioned in the introduction, in order to study the histograms of gold nanowires we have performed classical molecular dynamics (MD) simulations to investigate the formation of the contacts, and we have used a tightbinding model to calculate the conductance during the breaking of the atomic necks. It is worth stressing that the resulting numerical effort is considerable 47 . In what follows we explain the details of both theoretical techniques.

\section{A. Molecular dynamics simulations}

We analyze the breaking of Au nanocontacts by means of classical MD simulations. The forces and energies are calculated using semi-empirical potentials derived from the effective medium theory $\stackrel{48}{ }$, which successfully describe experimental bulk and surface properties 49 and have also been successfully used for simulating nanowires by Jacobsen et. al $31,32,34,65,76$.

Transmission electron microscopy ${ }^{50}$ shows that in the last stage of the stretching process, nanocontacts of $\mathrm{Au}$ are crystalline and atom rearrangements take place in such a way that one of the crystal directions [100], [110] or [111] lies in stretching direction of the wire, independent of the starting crystal orientation. For that reason our starting configuration is a perfect fcc-lattice of 112 atoms in [100] direction ( $\mathrm{z}$ direction) of length $2.65 \mathrm{~nm}$ with a cross-section of eight atoms. This central wire is attached at both ends to two slabs of 288 atoms each, which are kept fixed. We also performed simulations with starting configurations of perfect lattices in [110] (central wire: 108 atoms; each slab: 196 atoms) and in [111] direction (central wire: 240 atoms; each slab: 720 atoms). The stretching process is simulated by separating both 
slabs symmetrically at fixed distance in every time step. The resulting stretching velocity of $2 \mathrm{~m} / \mathrm{s}$ is much bigger than in the experiments, but it is small compared with the speed of sound in the material $(3000 \mathrm{~m} / \mathrm{s})$. Thus the wire can re-equilibrate between successive instabilities, although collective relaxation processes might be suppressed 15,29 . The Newtonian equations of motion of the wire atoms are integrated via the velocity Verlet algorithm ${ }^{51}$ with a time step of $1.4 \mathrm{fs}$. A Nosé-Hoover thermostat ${ }^{51}$ maintains the average temperature at 4.2 $\mathrm{K}$. We used periodic boundary conditions for the slabs in $\mathrm{z}$ direction and minimum image convention for the slabs perpendicular to the $\mathrm{z}$ direction ${ }^{52}$. Before the stretching process, every atom of the wire gets a randomly chosen velocity (that is why every nanocontact evolves a little bit different from stretching to stretching) and the wire is equilibrated for about $0.7 \mathrm{~ns}$ with periodic boundary conditions perpendicular to the $\mathrm{z}$ direction.

In order to test whether the conductance changes are correlated with atom rearrangements in the nanocontact, we calculate the radius of the minimum cross-section perpendicular to the stretching direction as defined in Ref. [28]. For this purpose, every atom is represented by a sphere with the volume of the elementary cell in the fcc-lattice. For a given configuration a slice with a width of about the interlayer distance ${ }^{53}$ is taken perpendicular to the stretching direction. Out of the volume of the atomic spheres overlapping with the slice, the radius of a cylinder which would fill the same volume in that slice is computed. The procedure is repeated along the whole nanocontact and the smallest radius is taken as the radius of the minimum cross-section of a given configuration.

Moreover, during the stretching process, every $1.4 \mathrm{ps}$ a configuration is recorded and the strain force of the nanocontact is computed following Finbow et al. 54 . Every 5.6 ps the corresponding conductance is calculated using the method described in the next subsection.

\section{B. Conductance calculations}

The computation of conductance in atomic systems of the size that we consider here, is out of the scope of present ab-initio methods. For this reason, we chose to use a tight-binding model based on the parameterization introduced in Ref. 55]. As mentioned in the introduction, the tight-binding models have been very successful in the description of the transport of metallic atomic contacts 10.11 . Our approach follows closely the one of Refs. 1011 , with the only difference that in this case we use a non-orthogonal basis, which introduces some minor changes as explained in Refs. 56 57.

The first step in our approach is the description of the electronic structure of the atomic contacts, which is done in terms of the following tight-binding Hamiltonian written in a non-orthogonal basis

$$
\hat{H}=\sum_{i \alpha, \sigma} \epsilon_{i \alpha} c_{i \alpha \sigma}^{\dagger} c_{i \alpha \sigma}+\sum_{i \alpha \neq j \beta, \sigma} v_{i \alpha, j \beta} c_{i \alpha, \sigma}^{\dagger} c_{j \beta, \sigma},
$$

where $i, j$ run over the atomic sites and $\alpha, \beta$ denote the different atomic orbitals, $\epsilon_{i \alpha}$ are the on-site energies and $v_{i \alpha, j \beta}$ are the hopping elements. Additionally, we need the overlaps between the different orbitals, $S_{i \alpha, j \beta}$. We take all these parameters from the bulk parameterization of Ref. 55], which is known to accurately reproduce the band structure of bulk materials. The atomic basis is formed by the 9 orbitals: $5 d, 6 s, 6 p$, which give rise to the main bands around the Au Fermi energy. It is important to emphasize that in this parameterization both, the hopping elements and the overlaps, are functions of the distance between the atoms, which allows us to apply it in combination with the MD simulations. These functions have a cut-off radius that encloses up to five nearest neighbors in a bulk geometry.

In an atomic contact the local environment in the neck region is very different to that of the bulk material. In particular, this fact can lead to large deviations from the approximate local charge neutrality that typical metallic elements exhibit. We correct this effect imposing local charge neutrality in all the atoms of the nanowire through a self-consistent variation of the diagonal parameters $\epsilon_{i \alpha}$.

The quantity that we are interested in is the lowtemperature linear conductance which can be expressed in terms of the Landauer formula

$$
G=G_{0} \operatorname{Tr}\left\{\hat{t}\left(E_{F}\right) \hat{t}^{\dagger}\left(E_{F}\right)\right\}=G_{0} \sum_{n} T_{n},
$$

where $\hat{t}$ is the transmission matrix of the contact, and the $T_{n}$ 's are the transmission eigenvalues at the Fermi energy $E_{F}$. The transmission matrix can be calculated in terms of the Green functions of the contact as follows

$$
\hat{t}(E)=2 \hat{\Gamma}_{L}^{1 / 2}(E) \hat{G}_{C}^{r}(E) \hat{\Gamma}_{R}^{1 / 2}(E) .
$$

Here, $\hat{\Gamma}_{L, R}$ are the scattering rate matrices given by $\hat{\Gamma}_{L, R}=\operatorname{Im}\left(\hat{\Sigma}_{L, R}\right)$, where $\hat{\Sigma}_{L, R}$ are the self-energies which contain the information of the electronic structure of the leads and their coupling to the central part of the contact. In our case the leads start in the slabs with 288 atoms and the central part, denoted by $C$, is the central wire. The self-energies can be expressed as

$$
\hat{\Sigma}_{L, R}=\left(\hat{v}_{C L, R}-E \hat{S}_{C L, R}\right) \hat{g}_{L, R}\left(\hat{v}_{L, R C}-E \hat{S}_{L, R C}\right),
$$

where $\hat{v}$ is the hopping matrix which describes the connection between the central cluster and the leads, and $\hat{S}$ is the corresponding overlap matrix. In this expression, $\hat{g}_{L, R}$ are the Green functions of the uncoupled leads, 


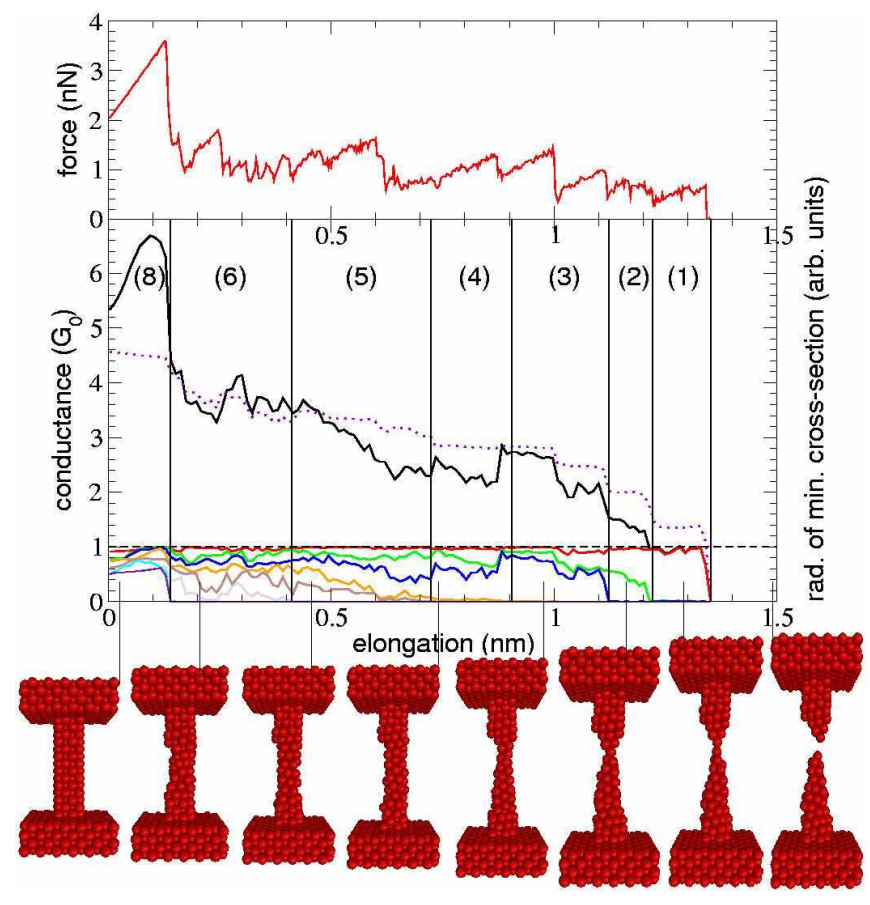

FIG. 1: (Color online). Formation of a dimer configuration of $\mathrm{Au}$ in the [100] direction at $T=4.2 \mathrm{~K}$. The upper panel show the strain forces as a function of the elongation of the contact. In the lower panel we show the conductance (solid line), the radius of the minimum cross-section (dashed line), and the channel transmissions (lines in the bottom of the graph). The vertical lines define regions with different number of open channels ranging from 8 to 1 . Below the graph we show snapshots of the breaking of the contact.

which we assume to be the bulk Green functions of $\mathrm{Au}$. Finally, the central cluster Green functions, $\hat{G}_{C}$, are given by

$$
\hat{G}_{C}(E)=\left[E \hat{S}_{C}-\hat{H}_{C}-\hat{\Sigma}_{L}(E)-\hat{\Sigma}_{R}(E)\right]^{-1},
$$

where $\hat{H}_{C}$ and $\hat{S}_{C}$ are the Hamiltonian and the overlap matrix of the central part, respectively.

\section{EXAMPLES OF THE CONTACT FORMATION}

In this section we discuss in detail some of the typical features that we observe in single realizations of the $\mathrm{Au}$ contacts. In particular, we discuss the formation of both single-atom contacts and chains.

\section{A. Single-atom contacts}

In Fig. 1 we show the formation of what we could call a single-atom contact. To give a complete information of this process we have depicted the forces, the conductance, the radius of the minimum cross-section, and the transmissions of the different conduction channels. In this example the conductance evolves during the elongation process from a value close to $5 G_{0}$ for the starting point to $1 G_{0}$ in the last stages before breaking ${ }^{58}$. The decrease of the conductance follows closely the evolution of the minimum cross-section. This correlation is particularly pronounced in the last stages when the contact has one or two atoms in the narrowest part. However, it is worth stressing that the conductance does not always follow the minimum cross-section, as can be seen in the elongation region between 0.75 and $1.0 \mathrm{~nm}$. Sometimes there appear jumps in the conductance in regions where the minimum cross-section evolves smoothly. We interpret this fact as rearrangements of atoms away from the narrowest part of the wire. This interpretation is confirmed by the analysis of the forces, as we explain in the next paragraph.

As shown in the upper panel of Fig. 1 one can see in the forces a series of regions where they increase linearly (elastic stages) and regions with abrupt jumps (plastic stages). The first ones correspond to situations where the structure remains fundamentally unchanged, and the latter correspond to the breaking of bonds and the subsequent sudden atomic rearrangements. As commented in the introduction, this evolution of the forces has been measured for $\mathrm{Au}$ contacts at room temperature with the help of an atomic-force microscope $\frac{16}{}$. The order of magnitude of the forces in Fig. [1 is in good agreement with this experiment. Notice that also in the cases where the jumps in the conductance are not correlated to abrupt changes in the minimum cross-section, one sees jumps in the forces. This indicates that plastic deformations in regions away from the narrowest part of the wire have also an influence on the conductance. Thus, we see that in the determination of the conductance, the minimum cross-section is an important ingredient, but it is by no means the only one. This fact will be crucial later on for the interpretation of the conductance histograms.

Another interesting feature in Fig. 1 is the last conductance plateau. It corresponds to a contact with the crosssection of one atom, but it is formed by a dimer of gold atoms. This dimer configuration is the most common geometry that we find in the last stages of the contact breaking. This type of configuration has been reported recently in $\mathrm{MD}$ simulations of the breaking of $\mathrm{Al}$ wires 59 . Notice also that in the last plateau, which is marked by a linear increase of the forces, the conductance is close to $1 G_{0}$ and it is clearly dominated by a single conduction channel. As explained in Refs. 10 1112, this is due to the fact that the number of channels is controlled by the number of valence orbitals in the narrowest part of the contact. In the case of $\mathrm{Au}$ the main contribution to the density of states at the Fermi energy comes from the $6 \mathrm{~s}$ band, i.e. there is a single valence orbital. This implies that in the dimer configuration of Fig. [1 there is only one possible path (conduction channel) which proceeds 
through the $s$ orbitals. It is also important to emphasize that during the elongation of the contact we observe that the channels disappear one by one in agreement with the experimental results reported in Ref. [12]. This will be discussed in more detail below. Let us point out that we consider a channel being closed if its transmission is below $0.01 G_{0}$. We attribute the successive closing of the channels to the fact that in the stretching process the $\mathrm{Au}$ atoms leave the narrowest part one by one, and every $\mathrm{Au}$ atom contributes to the transport with one orbita ${ }^{60}$, which in turn can give rise to one conduction channel.

\section{B. Atomic chains}

In some occasions we observe that the contact does not break as in the configuration of Fig. 1 but continues to form chains of several atoms. This is illustrated in Fig. 2] Experimental evidence of the formation of gold chains was first reported independently by two groups 45.46 . The formation of chains had been already suggested by different simulations 31.32 .54 . In the last years there has been an intense activity in this topic. Thus for instance, there are now several experiments confirming the existence of gold chains ${ }^{61.62 .63}$; chains have been also observed in other materials ${ }^{64}$, and the forces during the formation of a $\mathrm{Au}$ chain have been measured 65 . From the theoretical side, many authors have analyzed the formation, stability and conductance of gold

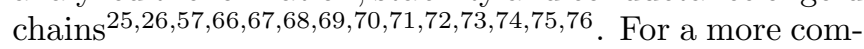
plete list of references, see section XI in Ref. [1]. We want to point out that so far there is no theoretical analysis of the conductance during the chain formation available.

In our simulations for the [100] direction we have observed 11 chains out of 50 stretching processes with different lengths: nine chains ranging from 3 atoms to 6 atoms and three chains of 10,12 and 14 atoms. Experimentally chains up to $8 \mathrm{Au}$ atoms have been reported ${ }^{46}$. The mechanism of the chain formation has been explained by Bahn and Jacobsen ${ }^{76}$ in terms of many-body effects in metals. The main idea is that in certain metallic systems the binding energy per neighboring atom may increase as the number of neighbors decreases. Here, we want to illustrate how a chain is formed in real time. We show in Fig. 3 five snapshots of the dynamics of the nanocontact of Fig. 2. At 0.0 ps an one-atom contact is shown with a typical dimer structure in the middle. The atom number 2 has four nearest neighbors and the atom 1 only three. Due to the many-body interactions the bond between the atoms 2 and 3 breaks. After about 40 ps another bond breaks and the atom 2 moves into the chain. The atom 4 is now in a similar situation as the atom 2 was at the beginning, and the previous process can be repeated again.

Turning now to the transport properties of the chains, we see in Fig. 2 that during the chain formation the conductance ranges from $0.6 G_{0}$ to $1.1 G_{0}$, exhibiting a long flat plateau close to $1 G_{0}$. With respect to the conductance value, this is in good agreement with experiment ${ }^{77}$.

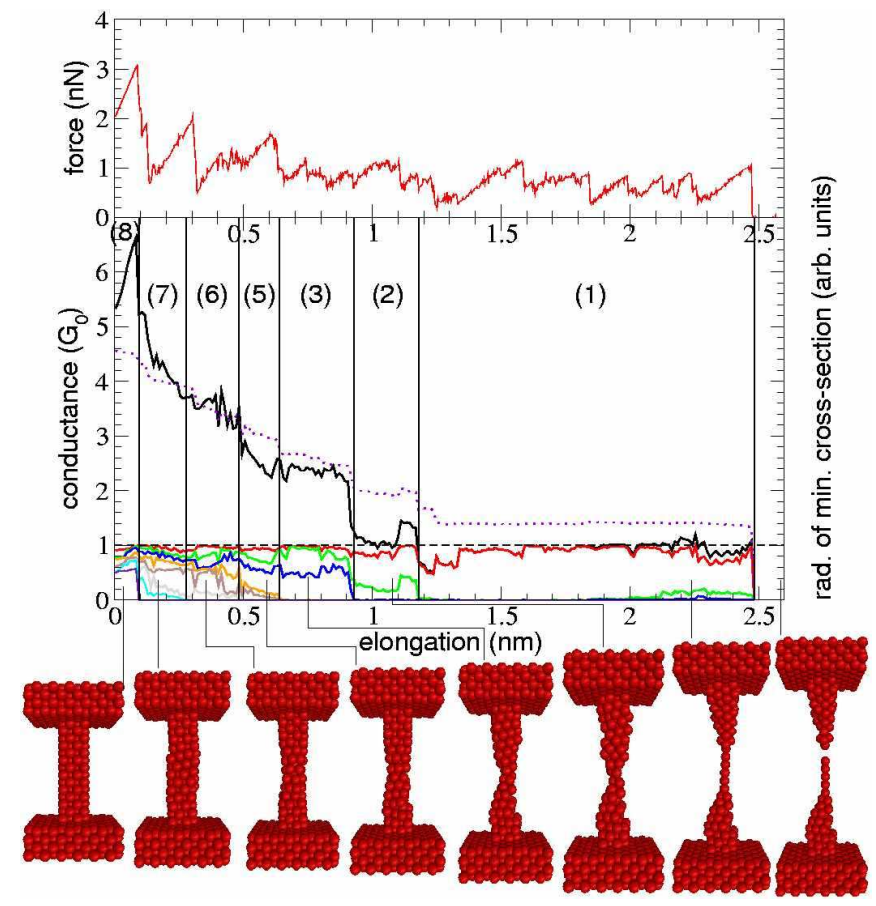

FIG. 2: (Color online). Formation of Au chain in the [100] direction at $T=4.2 \mathrm{~K}$. The upper panel show the strain forces as a function of the elongation of the contact. In the lower panel we show the conductance (solid line), the radius of the minimum cross-section (dashed line), and the channel transmissions (lines in the bottom of the graph). The vertical lines define regions with different number of open channels ranging from 8 to 1 . Below the graph we show snapshots of the breaking of the contact.

We observe that the conductance is mainly dominated by a single conduction channel. As in the case of a one-atom contact, the fact of having a single dominant channel is a consequence of the fact that $\mathrm{Au}$ is a monovalent metal. At the end of the stretching process, when the chain is made up of four or more atoms, we typically observe the appearance of a second channel and sometimes even a third one (see Fig. 2). Our analysis shows that these additional channels are due to the contribution of the $d$ orbitals. With respect to the fluctuations of the conductance during the formation of the chains, we want to stress that they are not an artifact of our conductance calculations, but they are clearly related to fluctuations in the contact geometry. In Fig. 22 one can see that the structure in the conductance during the chain formation is accompanied by abrupt changes in the force values, i.e. by plastic deformations, which correspond to the incorporation of another atom to the chain. We have calculated the conductance for all the configurations every $5.6 \mathrm{ps}$, including configurations that could be unstable. In the experiment the stretching velocity is several orders of magnitude smaller and such unstable configurations are averaged out. 


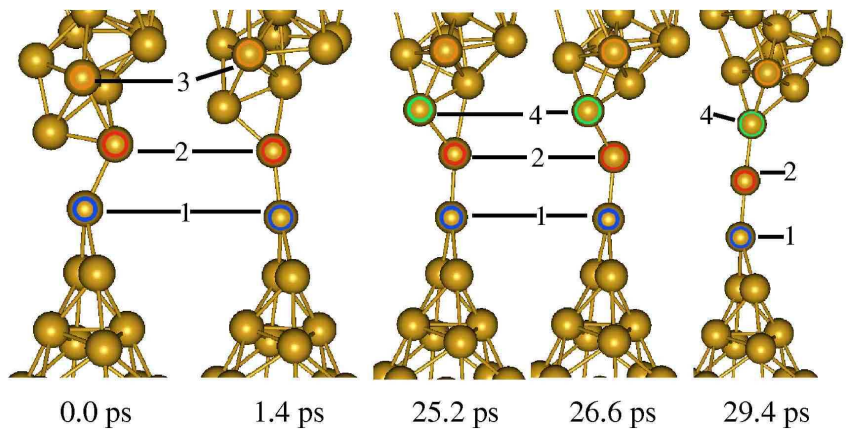

FIG. 3: (Color online). Snapshots of the chain formation of Fig. 2 At an initial time 0.0 ps the atom number 1 has three nearest neighbors and the atom 2 has four neighbors. At 1.4 ps the bond between atom 2 and atom 3 breaks. At 25.2 ps the atom 2 is only connected to two atoms of the electrode. At 26.6 ps another bond breaks and atom 4 is now in a similar environment as atom 2 at the beginning.
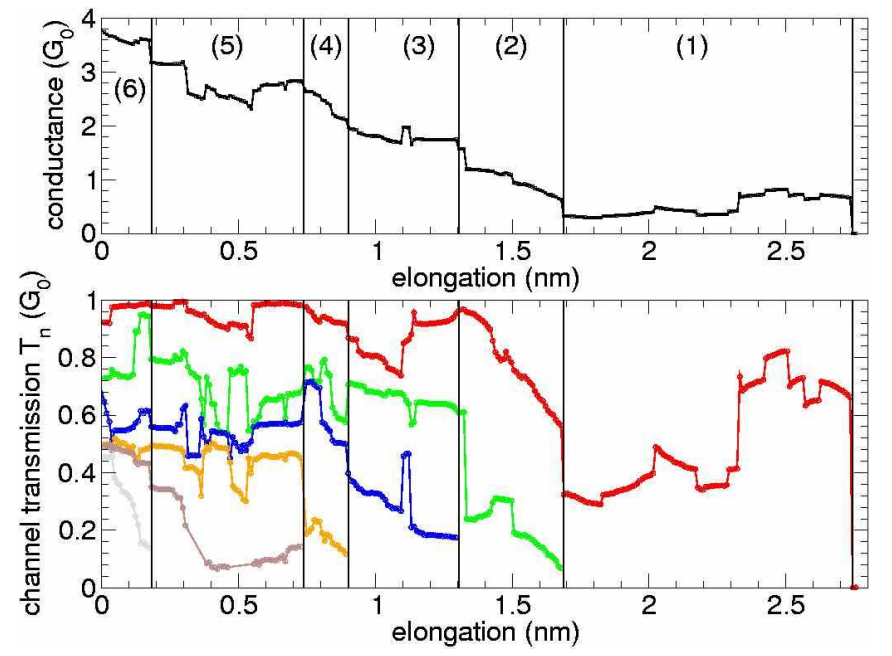

FIG. 4: (Color online). Measured total conductance of a Au sample (top panel) and single-channel contributions (bottom panel) as a function of the electrode distance $\frac{80}{\text { (opening }}$ curve). The vertical lines define regions with different numbers of channels ranging from 6 to 1 . The temperature was below $100 \mathrm{mK}$. For experimental details see Refs. [42, 43].

\section{COMPARISON WITH THE EXPERIMENTAL RESULTS}

In this section we show experimental results obtained following the technique of Refs. 12, 42, 43, where part of the data has been already presented. In these experiments it is possible to extract the full information of the individual transmission coefficients. This is done inducing proximity superconductivity in a Au contact and analyzing the super-conducting current-voltage characteristics 78.79 . We refer the reader to Ref. 43] for further details. Fig. 4 shows the conductance and the transmission coefficients of a Au contact as a function of the elon-
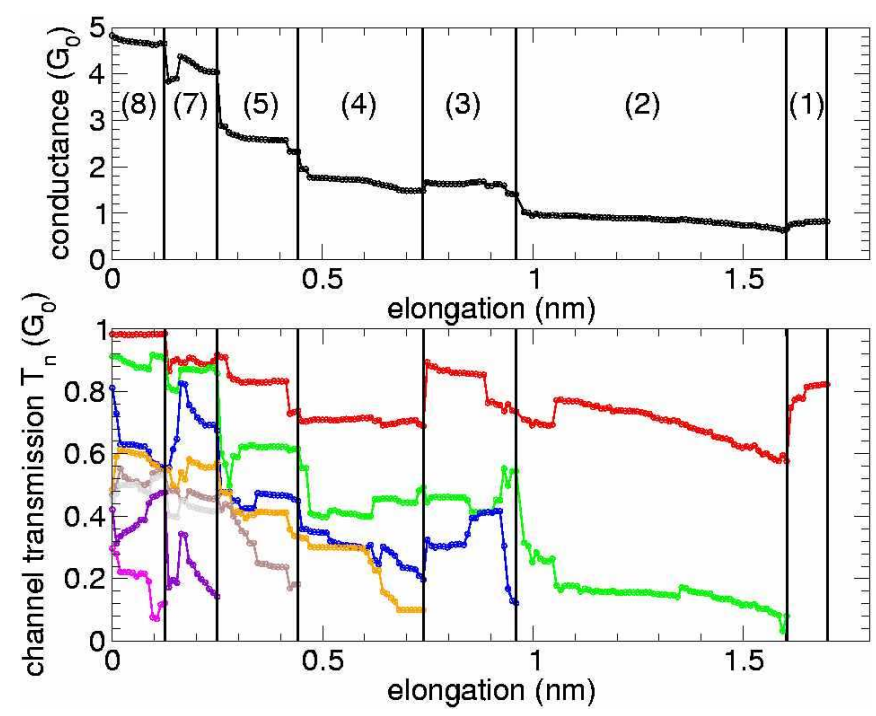

FIG. 5: (Color online). Measured total conductance of a Au sample (top panel) and single-channel contributions (bottom panel) as a function of the electrode distance ${ }^{80}$ (opening curve). The vertical lines define regions with different numbers of channels ranging from 8 to 1 . The temperature was below $100 \mathrm{mK}$. For experimental details see Refs. 42, 43].

gation at $100 \mathrm{mK}$. We can see how the channels disappear one by one as the contact evolves. Notice that there is a very long plateau of about $1 \mathrm{~nm}$ length, which corresponds to the formation of a chain. In this plateau the conductance changes between $0.3 G_{0}$ and $0.9 G_{0}$ and it is dominated by a single channel. Notice also the abrupt changes of the conductance, which are most likely due to incorporation of new atoms to the chain.

In Fig. 5 we show another experimental conductance curve. As it can be seen, there is a long plateau (about $0.7 \mathrm{~nm})$, where the conductance is close to $1 G_{0}$. Naively, one would expect the conductance to be dominated by a single conduction channel. However, the analysis reveals the presence of two channels, and only in the very last stages it reduces to one channel. In the two channel regime of the experiment in Fig. 5 the second channel has a transmission mainly below $0.2 G_{0}$ and the total conductance is slightly below $1 G_{0}$. In our simulations we typically observe two channels in the last stages for geometries in which there are two atoms in the narrowest part displaced with respect to each other (see Fig. 6(a)). If there is only a single atom, the second channel gives only a small contribution to the conductance (see Fig. 6(b)). As soon as we observe the dimer (see Fig. [6 (c)), the conductance is largely dominated by a single channel. Our analysis of the character of the channels suggests that for a single central atom like in Fig. 6(b) the second channel is due to direct tunneling between the electrodes. In the dimer configuration the distance between the electrodes is considerably larger, which leads to the disappearance of the second channel. The current then flows 


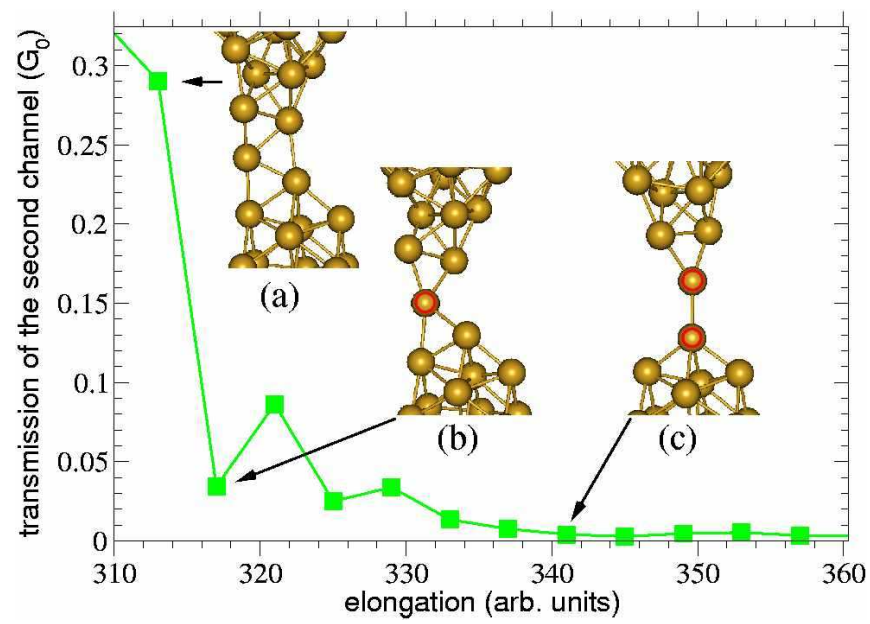

FIG. 6: (Color online). Transmission of the second channel during the last stages of the breaking of a typical Au contact in the $[100]$ direction $(\mathrm{T}=4.2 \mathrm{~K})$. The transmission of the first channel is about $0.7 G_{0}$.

only through the $s$ orbitals of the central atoms.

The connection between the atomic structure and the number of channels described above is supported by the experimental curve of Fig. 7 taken during the closing of a contact. In contrast to Fig. 4 and Fig. 5 and all the simulations, where the contacts were stretched (producing a so called opening curve), in Fig. [7 the two pieces of a nanocontact were brought into contact again after disruption (thereby producing a so called closing curve). It seems very unlikely to produce a dimer by reconnecting a teared nanocontact, since the atoms collapse back after rupture into the bulk. In fact, one expects a single atom coupled to the two pieces of the nanocontact as smallest contacts, i.e. one expects two channels at the beginning. Indeed, the typical closing curve (Fig. [7) starts with two open channels.

\section{MINIMUM CROSS-SECTION HISTOGRAM}

In order to discuss in more detail the mechanical properties and stability of the contacts we have calculated histograms of the radius of the minimum cross-section for the three main crystallographic directions of the fcclattice. The results are summarized in Fig. 8 In this figure we have normalized the radius to the radius of an ideal linear chain $R_{0}=1.268 \AA$ with nearest neighbor distance (lattice constant $/ \sqrt{2}$ ). The most prominent feature of the histograms is the presence of peaks, especially for the [100] direction. These peaks suggest the existence of exceptionally stable radii. This is relatively surprising at low temperatures $(4.2 \mathrm{~K})$, where the gold atoms do not have enough kinetic energy to explore many configurations in order to minimize the surface energy.

It is important to remark that these peaks generally

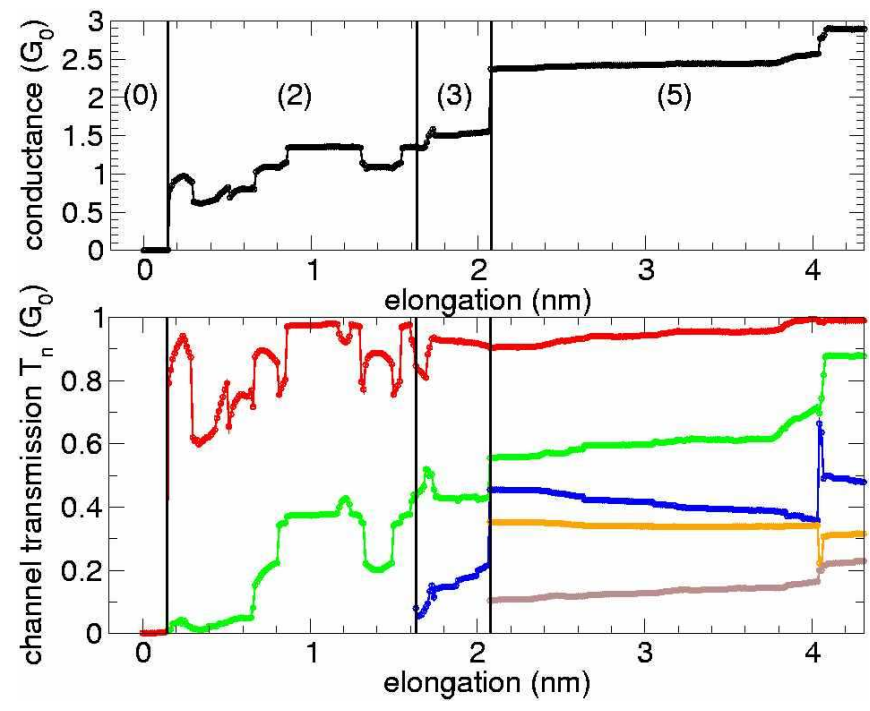

FIG. 7: (Color online). Measured total conductance of a $\mathrm{Au}$ sample (top panel) and single-channel contributions (bottom panel) as a function of the electrode distance ${ }^{80}$ (closing curve). The vertical lines define regions with different numbers of channels ranging from 2 to 5 . The temperature was below $100 \mathrm{mK}$. For experimental details see Refs. 42, 43].

do not appear at the values $\sqrt{n} \cdot R_{0}(n=1,2,3, \ldots)$, and therefore we cannot infer that the cross-sections associated with these peaks correspond to multiple of the cross-section of a single-atom contact. Furthermore in the three cases the peak close to 1 is extremely pronounced. This is due to the fact that usually in the last stages a dimer configuration or a chain of atoms is formed.

The next obvious question is whether this peak structure is an evidence of shell effects as observed for alkali metals $\frac{17}{17}$ and suggested more recently for gold contacts ${ }^{23.24}$. For shell effects the peaks are expected to be equidistant when plotted as a function of the radius $R$ of the wire ${ }^{17}$. To test this idea in the inset of Fig. 8 we plot $k_{F} R$ as a function of the peak number for the histogram of the [100] direction. Here, $k_{F}$ is the Fermi momentum, which is assumed to take the free-electron value of $1.21 \times 10^{8} \mathrm{~cm}^{-1}$. As one can see, the peak positions indeed follow a straight line with slope $0.60 \pm 0.02$. This value of the slope lies in between the values observed in Ref. 24] for electronic shells (1.02) and for atomic shells (0.40). From our analysis we cannot draw any conclusion on the appearance of shell effects in our simulations for several reasons. First, the choice of the peak positions is not absolutely unambiguous, and second, a similar analysis for the histograms in the directions [110] and [111] does not show such a clear linear relation between the radius and the peak position. It is worth stressing that even though the peak structure of these histograms cannot be easily interpreted in terms of shell effects, it clearly shows that particularly stable configurations do 

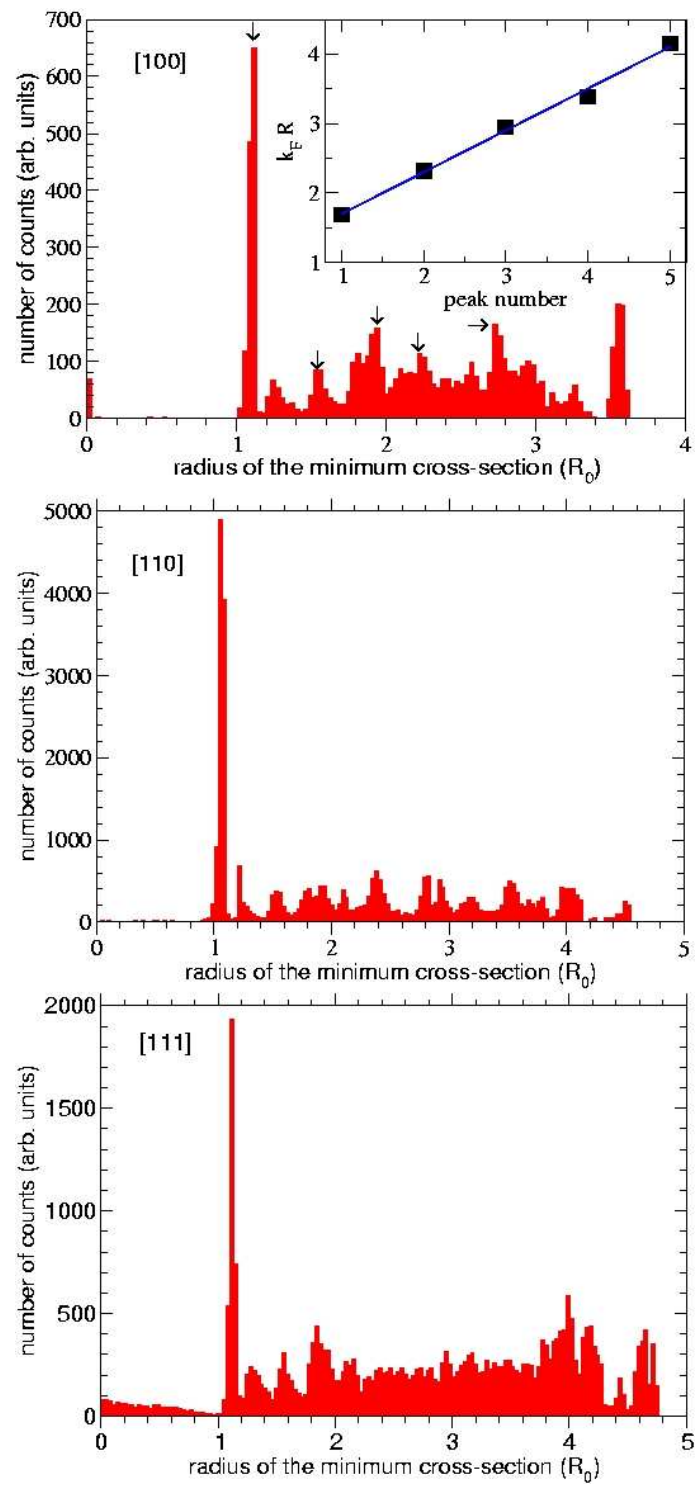

FIG. 8: (Color online). Histograms of the radius of the minimum cross-section $R$, which is normalized in units of the radius of an ideal linear chain of gold atoms $R_{0}=1.268 \AA$. The temperature is $4.2 \mathrm{~K}$. The different panels correspond to different crystallographic orientations. The number of simulations used to construct the histograms is 50 for each directions ${ }^{81}$. The inset in the upper panel shows the peak positions in the [100] histogram, converted to $k_{F} R$, as a function of the peak index. The arrows in the upper panel indicate the peak positions used to construct this plot. The last peak of the [100] histogram is artificially generated by the starting configurations.

exist, which most likely correspond to meta-stable geometries which are formed at low temperatures.

\section{CONDUCTANCE HISTOGRAM}

In this section we want to address the main issue of this work, namely the origin of the peaks in the conductance histograms. Are these peaks simply due to the peaks in the minimum cross-section histograms as suggested in Ref. 22]? In order to answer this question, we have collected the conductance calculations ${ }^{81}$ for all our MD simulations in the [100] direction at $4.2 \mathrm{~K}$. The results are displayed in the bottom panel of Fig. 9 . As one can see, the conductance histogram has a pronounced peak close to $1 G_{0}$ and two further maxima above $2 G_{0}$ and $3 G_{0}$. As we show below, the peak close to $1 G_{0}$ is highly correlated with the first peak in the corresponding minimum cross-section histogram of Fig. 9 (upper panel), and it is therefore a consequence of formation of dimer configurations or chains of atoms. However, the other peaks are not reflected in the conductance histogram in contrast to the postulated correlation between these two types of histograms for $\mathrm{Al}^{22}$. We explain this fact as follows. As we pointed out in section III, the minimum cross-section is not the only ingredient that controls the conductance, and the geometry of the narrowest part and the disorder in the contact play also an important role. This means in practice that contacts with different radii can have similar values of the conductance. In particular, this is true for small contacts like the one we have analyzed in this work. Of course, the situation could be different for larger contacts, where semi-classical arguments are believed to provide a good description.

Let us now illustrate the correlation between the conductance histogram and the minimum cross-section histogram. For this purpose, we show in Fig. 9 both histograms in a color/grey scale representation. The color/grey scale of the counts shows how many counts of a certain conductance value corresponds to a certain region of the radius histogram. Notice that the peak in the conductance histogram close to $1 G_{0}$ is clearly due to the contribution of contacts with the cross-section of an atom, i.e. single-atom contacts or chains of atoms. The other minimum cross-section regions overlap in the conductance histogram.

Another important piece of information can be obtained by analyzing the individual transmission coefficients. Fig. 10] shows the mean value ${ }^{82}$ of the channel transmissions $\left\langle T_{n}\right\rangle$ as a function of the total conductance for the conductance histogram of Fig. 9 Notice that below $1 G_{0}$ the conductance is clearly dominated by a single channel. In particular, for a total conductance of $1 G_{0}$ the second channel gives only a contribution about $0.036 G_{0}$. This is due to the fact that this region corresponds mainly to dimer configurations or chain of atoms, where all the current proceeds through the $6 s$ orbital of gold, as explained in section III.

In the two channel regime of the experiment in Fig. 15 the second channel has a transmission mainly below $0.2 G_{0}$ and the total conductance is slightly below $1 G_{0}$. Similar values are obtained in the simulations. For ex- 

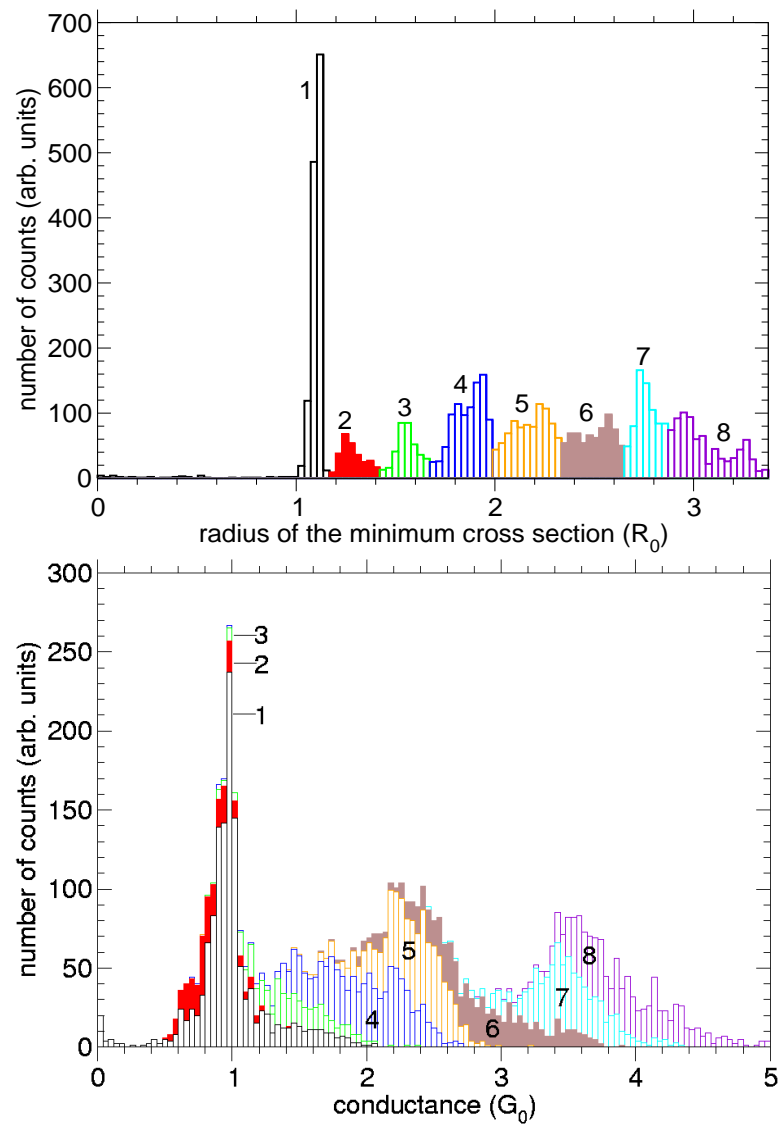

FIG. 9: (Color online). The lower panel shows the conductance histogram for $\mathrm{Au}$ in [100] direction for $T=4.2 \mathrm{~K}$. We focus on the region $G<5 G_{0}$ after the initial relaxation of the ideal contacts 58 . The color/grey scales indicate how many counts of a certain conductance value correspond to a certain region of the histogram of the radius of the minimum crosssection. This latter histogram is shown in the upper panel with the different regions defined ${ }^{83}$ with different color/grey scales. In order to make it easier to distinguish the different colors in grey scaled print the colors are numbered.

ample, in Fig. 10 we observe at a total mean conductance of about $1.1 G_{0}$, i.e. in the two channels regime (the third channel has only a mean transmission of under $0.03 G_{0}$ ), a mean transmission of the second channel of about $0.18 G_{0}$. For higher conductance values, the channels are not completely open, which explains the absence of conductance quantization in the histogram.

\section{CONCLUSIONS}

We have presented a comprehensive theoretical study of the mechanical and electrical properties of Au nanocontacts at low temperatures and provide a comparison with experimental results on the transport channels. Our main goal was to analyze how the interplay of these two type of properties is reflected in the conductance his-

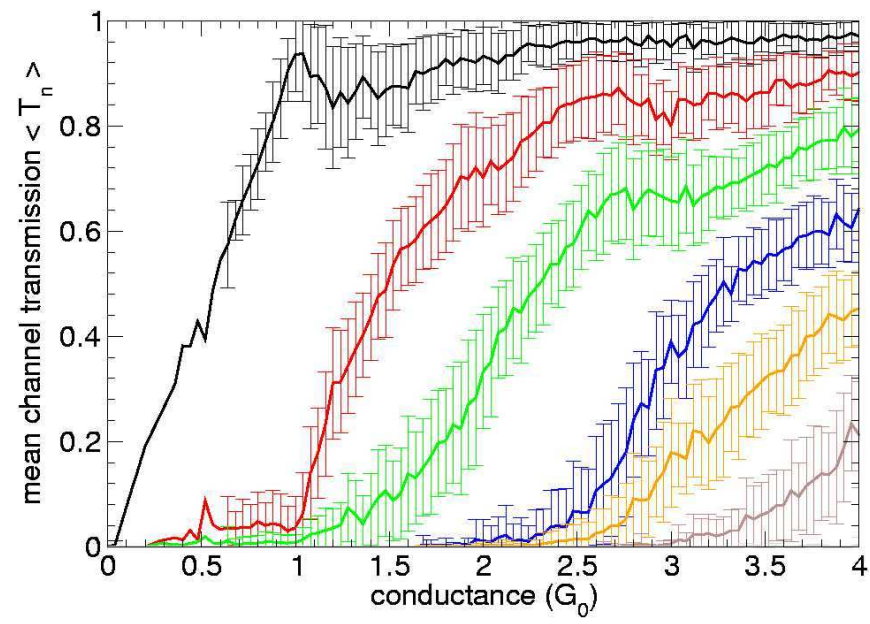

FIG. 10: (Color online). Mean value of the transmission coefficients $\left\langle T_{n}\right\rangle(n=1$ until $n=6)$ as a function of the conductance in units of $G_{0}$ for $\mathrm{Au}$ in [100] direction for $T=4.2 \mathrm{~K}$, i.e. corresponding to the histogram of Fig. 9 The error bars indicate the standard deviations of the numerical results (if enough data points are available).

tograms. We have shown that the histograms of the minimum cross-section show a peak structure, which suggests that there are contact configurations which are particularly stable. Semi-classical arguments, which are mainly based on the Sharvin formula or modifications of it, indicate that the conductance is only controlled by the ratio between the radius of the contact and the Fermi wave length. With these arguments one would expect to see also a series of peaks in the conductance histograms, which are correlated with the peaks of the histogram of the minimum cross-section. However, in our calculations this is not the case, and only the peak close to $1 G_{0}$ is clearly correlated with the first peak of the histogram of the minimum cross-section. In other words, we find that (i) the minimum cross-section is not the only ingredient that determines the conductance and (ii) the peak at $1 G_{0}$ is due to the formation of single-atom contacts and chains of atoms. Our theoretical conductance histogram is in qualitative agreement with the experimental findings at low temperatures. We note however, that we cannot expect more than qualitative agreements due to the different stretching process realization in the experiments and in the simulation.

We have also found the following interesting results. In our simulations the most typical geometry in the last stages of the breaking of the contact is a dimer configuration where two $\mathrm{Au}$ atoms are facing each other (see Fig. (1). Our analysis of the conduction channels shows that the conductance is dominated by a single channel in the last plateau, which corresponds to a single-atom contact or a chain of atoms. Moreover, the channels disappear one by one as the section of the contact is reduced. Both observations are due to the fact that $\mathrm{Au}$ is a monovalent metal, where every atom contributes with a single 
valence orbital to the transport. These finding are in good agreement with the experimental observations (see Fig. 45). Furthermore the simulations indicate a small contribution of the second channel to the total conductance if only one single atom is coupled to the electrodes. This finding can explain the number of channels of the opening curve of the experiment (see Fig. 7). In addition, as shown in Fig. 5 and in agreement with our simulations, the last conductance plateau in the experiment is not always carried by only one single channel, as is often assumed.

Of course, it would be desirable to extend our analysis to higher temperatures to investigate the appearance of shell effects 23.24 . On the other hand, it would be interesting to compare the results for $\mathrm{Au}$ with results for other materials, in particular multivalent ones, to explain the differences in the conductance histograms. Both type of studies are currently under progress.

\section{ACKNOWLEDGMENT}

It is a pleasure to thank M. Häfner for useful discussions. Support by the SFB 513 and granting of computer time from the NIC and the HLRS is gratefully acknowledged. FP and JCC were financially supported by the Landesstiftung Baden-Württemberg within the "Kompetenznetz Funktionelle Nanostrukturen" and by the Helmholtz Gemeinschaft within the Nachwuchsgruppe program (contract VH-NG-029). The measurements have partially been performed at CEA Saclay, France. PN thanks the institutes for theoretical physics of the FU Berlin and the U Mainz for the hospitality during his sabbatical stay.
1 N. Agraït, A. L. Yeyati and J.M. van Ruitenbeek, Phys. Rep. 377, 81 (2003).

2 C.J. Muller, J.M. van Ruitenbeek, and L.J. de Jongh, Phys. Rev. Lett. 69, 140 (1992).

3 N. Agraï, J.G. Rodrigo, and S. Vieira, Phys. Rev. B 47, 12345 (1993).

4 B.J. van Wees, H. van Houten, C.W.J. Beenakker, J.G. Williamson, L.P. Kouwenhoven, D. van der Marel, and C.T. Foxon, Phys. Rev. Lett. 60, 848 (1988); D.A. Wharam, T.J. Thornton, R. Newbury, M. Pepper, H. Ahmed, J.E.F. Frost, D.G. Hasko, D.C. Peacock, D.A. Ritchie, and G.A.C. Jones, J. Phys. C 21, L209 (1988).

${ }^{5}$ L. Olesen, E. Lægsgaard, I. Stensgaard, F. Besenbacher, J. Schiøtz, P. Stoltze, K.W. Jacobsen, and J.K. Nørskov, Phys. Rev. Lett. 74, 2147 (1995).

6 J.M. Krans, J.M. van Ruitenbeek, V.V. Fisun, I.K. Yanson and L.J. de Jongh, Nature 375, 767 (1995).

7 Z. Gai, Yi He, H. Yu, and W.S. Yang, Phys. Rev. B 53, 1042 (1996).

8 E. Scheer, P. Joyez, D. Esteve, C. Urbina and M.H. Devoret, Phys. Rev. Lett. 78, 3535 (1997).

9 A.I. Yanson and J.M. van Ruitenbeek, Phys. Rev. Lett. 79, 2157 (1997).

10 J.C. Cuevas, A. L. Yeyati and A. Martín-Rodero, Phys. Rev. Lett. 80, 1066 (1998).

11 J.C. Cuevas, A. L. Yeyati, A. Martín-Rodero, G. Rubio, C. Untiedt and N. Agraït, Phys. Rev. Lett. 81, 2990 (1998).

12 E. Scheer, N. Agraï, J.C. Cuevas, A. L. Yeyati, B. Ludoph, A. Martín-Rodero, G. Rubio, J.M. van Ruitenbeek and C. Urbina, Nature 394, 154 (1998).

13 U. Landman, W.D. Luedtke, N.A. Burnham, and R.J. Colton, Science 248, 454 (1990).

14 A.P. Sutton and J. B. Pethica, J. Phys.: Condens. Matter 2, 5317 (1990).

15 T.N. Todorov and A.P. Sutton, Phys. Rev. Lett. 70, 2138 (1993).

16 G. Rubio, N. Agraït and S. Vieira, Phys. Rev. Lett. 76, 2302 (1996)

17 A. I. Yanson, I. K. Yanson, and J.M. van Ruitenbeek, Nature 400, 144 (1999); Phys. Rev. Lett. 84, 5832 (2000).
18 A. I. Yanson, I. K. Yanson, and J.M. van Ruitenbeek, Phys. Rev. Lett. 87, 216805 (2001).

19 C.A. Stafford, D. Baeriswyl and J. Bürki, Phys. Rev. Lett. 79, 2863 (1997)

20 J.M. van Ruitenbeek, M.H. Devoret, D. Esteve, and C. Urbina, Phys. Rev. B 56, 12566 (1997).

21 C. Yannouleas and U. Landman, J. Phys. Chem. B 101, 5780 (1997).

22 A. Hasmy, E. Medina, and P. A. Serena, Phys. Rev. Lett. 86, 5574 (2001)

23 E. Medina, M. Díaz, N. León, C. Guerrero, A. Hasmy, P. A. Serena, and J. L. Costa-Krämer, Phys. Rev. Lett. 91, $026802(2003)$.

24 A.I. Mares, A.F. Otte, L.G. Soukiassian, R.H.M. Smit, and J.M. van Ruitenbeek, Phys. Rev. B 70, 073401 (2004).

25 P.Z. Coura, S.B. Legoas, A.S. Moreira, F. Sato, V. Rodrigues, S.O. Dantas, D. Ugarte, and D.S. Galvão, Nanoletters 4, 1187 (2004).

26 E.Z. da Silva, F.D. Novaes, A.J.R. da Silva, and A. Fazzio, Phys. Rev. B 69, 115411 (2004).

27 J.A. Torres and J.J. Sáenz, Phys. Rev. Lett. 77, 2245 (1996); T. López-Ciudad, A. Garcia-Martin, A. J. Caamano, J. J. Saenz, Surf. Sci. 440, L887 (1999); J. Bürki, C. A. Stafford, X. Zotos, D. Baeriswyl, Phys. Rev. B 60, 5000 (1999). A. Garcia-Martin, M. del Valle, J.J. Sáenz, J.L. Costa-Krämer, and P.A. Serena, Phys. Rev. B 62, 11139 (2000).

28 A.M. Bratkovsky, A.P. Sutton and T.N. Todorov, Phys. Rev. B 52, 5036 (1995).

29 T.N. Todorov and A.P. Sutton, Phys. Rev. B 54, 14234 (1996).

30 H. Mehrez and S. Ciraci, Phys. Rev. B 56, 12632 (1997).

31 M. Brandbyge, M.R. Sørensen and K.W. Jacobsen, Phys. Rev. B 56, 14956 (1997).

32 M. R. Sørensen, M. Brandbyge, and K. W. Jacobsen, Phys. Rev. B 57, 3283 (1998).

33 A. Buldum, S. Ciraci, I. P. Batra, Phys. Rev. B 57, 2468 (1998).

34 A. Nakamura, M. Brandbyge, L. B. Hansen, and K. W. Jacobsen, Phys. Rev. Lett. 82, 1538 (1999). 
${ }^{35}$ V. Rodrigues, T. Fuhrer, and D. Ugarte, Phys. Rev. Lett. 85, 4124 (2000).

36 L.G.C. Rego, A.R. Rocha, V. Rodrigues, and D. Ugarte, Phys. Rev. B 67, 045412-1 (2003).

37 C.J. Muller, J.M. Krans, T.N. Todorov and M.A. Reed, Phys. Rev. B 53, 1022 (1996).

38 J.L. Costa-Krämer, Phys. Rev. B 55, R4875 (1997).

39 K. Hansen, E. Lægsgaard, I. Stensgaard, and F. Besenbacher, Phys. Rev. B 56, 2208 (1997).

40 C.Z. Li and N.J. Tao, Appl. Phys. Lett. 72, 894 (1998).

41 B. Ludoph and J.M. van Ruitenbeek, Phys. Rev. B 61, 2273 (2000).

42 E. Scheer, W. Belzig, Y. Naveh, M. H. Devoret, D. Esteve, and C. Urbina, Phys. Rev. Lett. 86, 284 (2001).

43 E. Scheer, W. Belzig, Y. Naveh, and C. Urbina, Adv. in Solid State Phys. 42, 107 (2002).

${ }^{44}$ G. Rubio-Bollinger, C. de las Heras, E. Bascones, N. Agraï, F. Guinea, and S. Vieira, Phys. Rev. B 67, 121407 (2003).

45 H. Ohnishi, Y. Kondo and K. Takayanagi, Nature (London) 395, 780 (1998).

46 A.I. Yanson, G. Rubio-Bolinger, H.E. van den Brom, N. Agraï, and J.M. van Ruitenbeek, Nature (London) 395, 783 (1998).

47 A typical computation of the structural evolution requires a computation of $1.1 \cdot 10^{6} \mathrm{MD}$ steps, where after the equilibration of the system every $4 \cdot 10^{3} \mathrm{MD}$ steps the conductance is calculated. The resulting numerical effort is about 400 CPU-hours (Intel Xeon CPU $2.80 \mathrm{GHz}$ ) for a single stretching process. The total CPU-time of our studies was about $20 \cdot 10^{3}$ CPU-hours.

${ }^{48}$ K.W. Jacobsen, P. Stoltze, J.K. Nørskov, Surf. Sci. 366, 394 (1996).

49 P. Stoltze, J. Phys.: Condens. Matter 6, 9495 (1994).

50 V. Rodrigues, T. Fuhrer, D. Ugarte, Phys. Rev. Lett. 85, 4124 (2000).

51 D. Frenkel and B. Smit, Understanding Molecular Simulation, Academic Press, 1996 and references therein.

52 M.P. Allen and D.J. Tildesley, Computer Simulation of Liquids, Oxford Science Publications, 1987.

53 The width of the slice is $a / 2$ for the [100] direction, where $a$ is the lattice constant, $a /(2 \sqrt{2})$ for the [110] direction, and $a / \sqrt{3}$ for the [111] direction.

54 G.M. Finbow, R.M. Lynden-Bell, I.R. McDonald, Molecular Physics 92, 705 (1997).

55 M.J. Mehl and D.A. Papaconstantopoulos, Phys. Rev. B 54, 4519 (1996).

56 E. Emberly and G. Kirczenow, Phys. Rev. Lett. 81, 5205 (1998).

57 M. Brandbyge, N. Kobayashi, and M. Tsukada, Phys. Rev. B 60, 17064 (1999).

58 We observe in all the simulations an increase of the conductance during the first stages of the stretching process, which is due to relaxation of the ideal fcc starting geometry. Only after this relaxation, an evolution characteristic of every single contact begins.

59 P. Jelínek, R. Pérez, J. Ortega, and F. Flores, Phys. Rev. B 68, 085403 (2003).

${ }^{60}$ For the starting cross-section with eight atoms one observes eight channels.
61 T. Kizuka, Phys. Rev. Lett. 81, 4448 (1998).

62 V. Rodrigues and D. Ugarte, Phys. Rev. B 63, 073405 (2001).

63 Y. Takai, T. Kawasaki, Y. Kimura, T. Ikuta, and R. Shimizu, Phys. Rev. Lett. 87, 106105 (2001).

64 R.H.M. Smit, C. Untiedt, A.I. Yanson, and J.M. van Ruitenbeek Phys. Rev. Lett. 87, 266102 (2001).

65 G. Rubio-Bollinger, S. R. Bahn, N. Agraït, K. W. Jacobsen, and S. Vieira Phys. Rev. Lett. 87, 026101 (2001).

66 J.A. Torres, E. Tosatti, A. Dal Corso, F. Ercolessi, J.J. Kohanoff, F.D. Di Tolla, and J.M. Soler, Surf. Sci. Lett. 426, L441 (1999).

67 D. Sánchez-Portal, E. Artacho, J. Junquera, P. Ordejón, A. García, and J. M. Soler Phys. Rev. Lett. 83, 3884 (1999).

68 M. Okamoto and K. Takayanagi , Phys. Rev. B 60, 7808 (1999)

69 H. Häkkinen, R.N. Barnett, and U. Landman , J. Phys. Chem. B 103, 8814 (1999).

70 E.G. Emberly and G. Kirczenow, Phys. Rev. B 60, 6028 (1999).

71 L. De Maria and M. Springborg, Chem. Phys. Lett. 323, 293 (2000).

72 H. Häkkinen, R.N. Barnett, A.G. Scherbakov, and U. Landman, J. Phys. Chem. B 104, 9063 (2000).

73 E.Z. da Silva, A.J.R. da Silva, and A. Fazzio, Phys. Rev. Lett. 87, 256102 (2001).

74 J.J. Palacios, A.J. Pérez-Jiménez, E. Louis, E. SanFabián, and J. A. Vergés, Phys. Rev. B 66, 035322 (2002).

75 Y.J. Lee, M. Brandbyge, M.J. Puska, J. Taylor, K. Stokbro, and R.M. Nieminen, Phys. Rev. B 69, 125409 (2004).

76 S. R. Bahn and K.W. Jacobsen, Phys. Rev. Lett. 87, 266101 (2001).

77 R.H.M. Smit, C. Untiedt, G. Rubio-Bollinger, R. C. Segers, and J. M. van Ruitenbeek Phys. Rev. Lett. 91, 076805 (2003).

78 D. Averin and A. Bardas, Phys. Rev. Lett. 75, 1831 (1995).

79 J.C. Cuevas, A. Martín-Rodero and A. L. Yeyati, Phys. Rev. B 54, 7366 (1996).

80 The absolute value of the electrode distance is arbitrary, only relative distances could be measured. In the bottom panel all the highest measured transmissions (circles) of each electrode distance are connected with straight lines, then the second highest transmissions of each electrode distance are connected and so on. This mapping of single contributions of the transmission to certain channels cannot be measured experimentally.

81 The convergence of the minimum cross-section histograms and the conductance histogram was checked by comparing the normalized histograms constructed with 10, 20, 30, 40 and 50 stretching processes.

82 For a certain value of the total conductance the channel contribution of the $n$th channel (up to $n=8$ channels were observed in the simulations) of all 50 stretching processes are added and then divided by 50 . This gives the mean value $\left\langle T_{n}\right\rangle$ for the considered total conductance value.

83 The number of the regions of the minimum cross-section histogram is a little bit arbitrary. However, if one defines instead of eight regions for example 21 (smaller) regions, the qualitative conclusions remain the same. 